\title{
Online Noninvasive Localization of Accessory Pathways in the EP Lab
}

\author{
Michael Seger ${ }^{1 \star}$, Gerald Fischer ${ }^{1}$, Robert Modre ${ }^{1}$, Bernhard Pfeifer ${ }^{1}$, \\ Friedrich Hanser ${ }^{1}$, Christoph Hintermüller ${ }^{1}$, Florian Hintringer ${ }^{2}$, \\ Franz Xaver Roithinger ${ }^{2}$, Thomas Trieb ${ }^{3}$, Michael Schocke ${ }^{3}$, and \\ Bernhard Tilg ${ }^{1}$
}

\footnotetext{
1 Institute for Biomedical Signal Processing and Imaging, University for Health Sciences, Medical Informatics and Technology, Innsbruck, Austria,

2 Department of Cardiology, Medical University Innsbruck, Austria

3 Department of Radiology I, Medical University Innsbruck, Austria michael.seger@umit.at, http://imsb.umit.at
}

\begin{abstract}
Inverse electrocardiography has been developing during the last years and is about to become a valuable clinical tool for analyzing arrhythmias and electrical dysfunction in general. By combining measurements obtained by electrocardiographic body surface mapping with three-dimensional anatomical data, the electrical activation sequence of the individual human heart can be imaged noninvasively. This technique was applied on-line in the electrophysiology lab. The results of four patients, who underwent an interventional electrophysiology study, are presented in this paper. The sites of early activation were compared to the locations of successful radio-frequency ablation. The location error was found to be between 13 and $20 \mathrm{~mm}$. This promising finding may bring this noninvasive method closer to clinical application.
\end{abstract}

\section{Introduction}

Atrial and ventricular arrhythmias are of great concern in clinical electrocardiology [12. In clinical practice, the localization of the origins of arrhythmias is currently achieved by traditional catheter techniques and by catheter mapping techniques 113. These techniques show significant limitations when it comes to acquiring single-beat activation maps. In addition, the invasive procedures cannot be used for screening patients in order to decide the optimal individual therapeutic procedure (e.g., drug, ablative or hybrid therapy). The simultaneous acquisition of three-dimensional (3D) anatomical and electrocardiographic (ECG) data of individual patients enables noninvasive imaging of the electrical function in the human heart. Three-dimensional anatomical data from the human thorax can be obtained, e. g., from magnetic resonance imaging (MRI). ECG mapping data can be acquired from the patient's chest surface with multichannel (32 up to 256 channels) biopotential recording systems. The coupling of

\footnotetext{
* Corresponding author 
these two modalities in time and space permits the imaging of electrical function when applying bioelectromagnetical field theory and solving an ill-posed inverse problem 4567.7. The primary electrical source in the cardiac muscle is the spatio-temporal distribution of the transmembrane potential (TMP) $\varphi_{m}[458$ 9. The potential on the chest surface and the potential on all other conductivity interfaces are related to $\varphi_{m}$ by a Fredholm integral equation of second kind. The relationship between the impressed electrical sources and the potentials at the electrode sites on the torso surface is described by the leadfield-matrix, the solution of the forward problem of electrocardiography [4,10]. The boundary element method (BEM) - a surface integral equation approach - is, in general, applied to solve this forward problem 4511. In the electrocardiographic inverse problem, the impressed electrical source distribution is estimated from the chest surface ECG mapping data. This imaging technique requires an electrical source model. Because of the generally unknown individual fiber orientation, electrical isotropy is assumed in the surface heart model approach based on the bidomain theory [5] 6.7. The most established inverse formulations are the imaging of the activation time (AT) map on the entire surface of the atria or ventricles and the imaging of the epicardial potential pattern [6]12. With the AT imaging approach, the time of onset of depolarization at each source node is estimated, whereas the epicardial potential problem aims at estimating the (extracellular) potential on the pericardium. When used in a straightforward manner, the epicardial potential formulation does not allow the imaging of the potentials on the endocardia. The heart model approach employing the bidomain theory, however, is capable of imaging the electrical excitation on the entire atrial or ventricular surface, i. e., on the epicardia as well as on the endocardia. This is of particular interest from a clinical point of view, as most of the catheter interventions in the electrophysiology (EP) lab are performed on the endocardia. The difference of this work to previous studies is, that the noninvasive imaging of cardiac electrical function was performed on-line in the EP lab. In former publications, e.g., in 6713, the computation of the AT was performed off-line and after the intervention. In order to demonstrate that noninvasive imaging of cardiac electrical function is feasible under clinical conditions, we applied the AT imaging approach on-line in the catheter laboratory: During the diagnostic part of the EP study using the electroanatomical mapping system CARTO $^{\mathrm{TM}}$ (Biosense Webster Inc.), the AT sequences were computed. The CARTO ${ }^{\mathrm{TM}}$ maps were used as 'gold standard' for the resulting AT maps. In this study, the on-line imaging of ventricular preexcitation was investigated as an important example for clinical application.

\section{Methods}

Four patient:1 (patient A - D; age: 19 - 35 years, 2 female) suffering from WolffParkinson-White (WPW) syndrome were included in the on-line study.

\footnotetext{
${ }^{1}$ Written informed consent was obtained from the patients before any diagnostic and therapeutic treatment. The study protocol was approved by the local ethics committee.
} 
The time schedule for the on-line procedure was organized as follows: Previous to the intervention, the patients were moved to the MRI scanner. Afterwards, the volume conductor model consisting of the lungs, chest, blood masses and the ventricles was generated. In the EP lab, the electrodes were attached on the patients' chest, the locations of the electrodes were digitized and the body surface potentials (BSP) were recorded. Before intervention, the on-line AT map was computed for a couple of single beats. After the diagnostic part with the

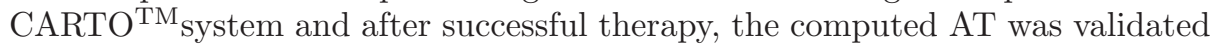
with the CARTO ${ }^{\mathrm{TM}}$ solution.

\subsection{Preprocessing}

MRI scanning. An MRI scan of the patients was made previous to the intervention. Individual anatomical data were obtained from MRI using a MagnetomVision-Plus 1.5 T (Siemens Medical Solutions) scanner. The ventricular geometry was recorded in CINE-mode during breath-hold (expiration, short-axis scans, $6 \mathrm{~mm}$ spacing). The shape of the lungs and the torso were recorded in $\mathrm{T} 1-$ FLASH-mode during breath-hold (expiration, long-axis scans, $10 \mathrm{~mm}$ spacing). Seven markers (vitamin E-capsules, anatomical landmarks on the anterior and lateral chest wall) were used to couple the locations of the electrodes to the MRI frame. Eleven capsules were attached on the back, in order to tag the positions of the posterior electrodes, which were not accessible during the EP study.

Generation of the Volume Conductor Model. The volume conductor model containing the relevant compartments (i.e., atria or ventricles, chest, lungs, blood masses) of the individual patient was generated using the software package amiraDev ${ }^{\mathrm{TM}} 3.0$ (TGS Europe Inc.) in combination with a semiautomatic myocardial extraction tool [14]. The lungs and chest could be segmented in a straightforward manner. A marching cubes algorithm was applied to the segmented compartments, which resulted in a triangulated surface mesh of the whole volume conductor model. The volume conductor model of patient $\mathrm{D}$ is depicted in Fig. 1,

\subsection{Online Procedure}

When the patient arrived in the EP lab, the posterior, lateral and anterior electrodes were attached on the patient's chest. The positions of the anterior and lateral electrodes were digitized using the Fastrak system (Polhemus Inc.). Additionally, the locations of the 7 anatomical landmarks were digitized in order to allow transformation into the MRI frame. The computation of the leadfieldmatrix and of the ATs, respectively, was performed on an INTEL $^{\mathrm{R}}$ Xeon $^{\mathrm{TM}}$ dual processor (2.8 $\mathrm{GHz}$ with $2 \mathrm{~GB}$ memory for each processor).

Computation of the leadfield-matrix. After transformation of the electrode coordinates into the MRI frame, the leadfield-matrix was computed. The leadfield-matrix $\mathbf{L}$ with dimension e $\times$ s describes the relationship between the impressed electrical sources and the (extracellular) potentials $\boldsymbol{\Phi}$ in e electrodes for all time steps $\mathrm{T}$ [4|10]:

$$
\mathbf{\Phi}=\mathbf{L} \boldsymbol{\Phi}_{m}
$$




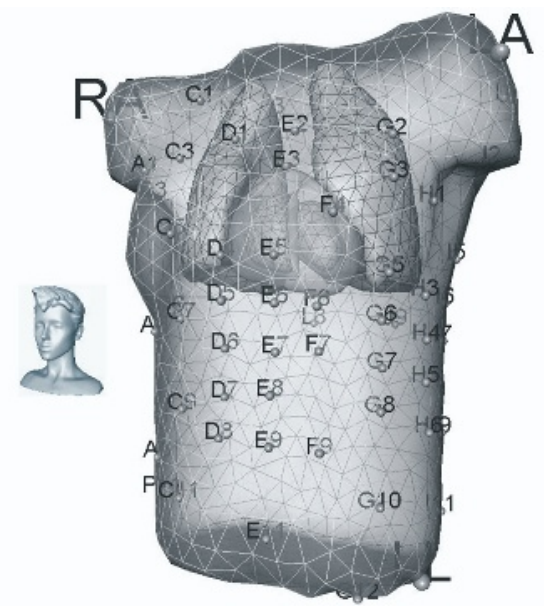

Fig. 1. Volume conductor model of patient D. The ventricles are shown in a transparent style, the blood masses and lungs are outlined. The electrodes are shown with their notation. The WCT defining the reference potential comprises the left arm (LA), right arm (RA) and left leg (LL). Note the head icon for spatial orientation.

with the s $\times$ T matrix $\boldsymbol{\Phi}_{m}$ containing the TMPs (electrical sources) in all s nodes of the cardiac surface. Note that $\boldsymbol{\Phi}_{m}$ is the time-discretized expression of $\varphi_{m}$. The computation of $\mathbf{L}$ was performed applying the BEM. The chosen conductivity values for the different compartments were chosen according to 4]: ventricles: effective intracellular conductivity: $0.1 \mathrm{Sm}^{-1}$, bulk conductivity: $0.2 \mathrm{Sm}^{-1}$; lungs: $0.08 \mathrm{Sm}^{-1}$, blood masses: $0.6 \mathrm{Sm}^{-1}$ and chest: $0.2 \mathrm{Sm}^{-1}$.

Acquisition of the BSP Map. The ECG mapping data was recorded before, during and after the EP study. The BSP map was acquired in 62 channels by the Mark-8 system (Biosemi V.O.F.). The Wilson-Central-Terminal (WCT) defined the reference potential [15. The sampling rate was $2048 \mathrm{~Hz}$. Signals were bandpass filtered with a lower and upper edge frequency of 0.3 and $400 \mathrm{~Hz}$, respectively. The AC-resolution of the system was $500 \mathrm{nVbit}^{-1}$ (16 bits per channel).

Signal preprocessing. For estimating the AT pattern, the ECG signals representing the depolarization sequences (target ECG waves) were used as input for further computation. In case of imaging ventricular pre-excitation, the QRS complex represents the target wave. The QRS complex was detected manually. The extracted signals were baseline corrected and no additional filtering was applied. Time discretization was set $1 \mathrm{~ms}$.

Estimation of the AT on the Heart Surface. The estimation of the ATs by considering (11) and (2) leads to an ill-posed, nonlinear (the TMP $\varphi_{m}(t)$ depends nonlinearly on the AT $\tau[6]$ ), inverse problem. Because of the ill-posedness, regularization techniques have to be employed in order to guarantee a stable solution in the presence of modeling errors and measurement noise. As the AT imaging 
focuses on the estimation of the time of onset of depolarization, the time course of the TMP at each source point of the ventricular (or atrial) surface can be approximated employing the analytical formula [6]

$$
\varphi_{m}(\tau, t)=\frac{u}{2}\left\{1+\frac{2}{\pi} \arctan \left[\pi \frac{t-\tau}{w}\right]\right\}+a,
$$

where a represents the resting transmembrane potential $(\mathrm{a}=-90 \mathrm{mV}), u$ the action potential amplitude $(\mathrm{u}=100 \mathrm{mV})$ and $\mathrm{w}$ the rise time $\mathrm{w}=2 \mathrm{~ms}$. Equation (2) determines the TMP time course and can therefore be considered as temporal regularization. Spatial regularization is achieved by adding the surface Laplacian [16] in the cost function [67], which is to be optimized with respect to $\tau$

$$
\left\|\mathbf{L} \Phi_{m}-\mathbf{D}\right\|_{F}^{2}+\lambda^{2}\|\Delta \tau\|^{2} \longrightarrow \min
$$

The surface Laplacian $\Delta$ is introduced in (3) in order to avoid an unphysiological AT pattern by smoothing the solution of the AT map. The parameter $\lambda$ determines the amount of regularization and is calculated employing the $L$-curve method 17, weighting the residual norm on the left hand side of (3) against the spatial regularization term. The $\mathrm{e} \times \mathrm{T}$ matrix $\mathbf{D}$ contains the measured ECG data in the electrodes and $\|\cdot\|_{F}$ represents the Frobenius norm. The interval $[0$, $\mathrm{T}$ ] reflects the duration of depolarization of the underlying target ECG wave. A Quasi-Newton solver was used for minimizing the functional in (3).

Validation of the computed AT map. The computed AT map was compared with the standard ECG diagnosis approach and with the CARTO ${ }^{\mathrm{TM}}$ map, respectively. After the intervention had been finished, the position of the 7 anatomical markers were digitized with the ablation catheter, in order to transform the $\mathrm{CARTO}^{\mathrm{TM}}$ map and the catheter ablation points into the MRI frame.

\section{Results}

The following is given for patient C. Similar computation and handling times were observed for all other patients. The MRI scanning took $1 \mathrm{~h} 5 \mathrm{~min}$, the segmentation of the volume conductor model was in the order of $1 \mathrm{~h} 15 \mathrm{~min}$. Another $23 \mathrm{~min}$ were used for attaching the electrodes and for digitizing their locations. Within 11 min the leadfield-matrix was calculated and two ECG sequences were recorded. After $4 \mathrm{~min}$ of signal preprocessing (determination of the target waves, baseline correction), the AT map was available after additional $11 \mathrm{~min}$. In case of patient $\mathrm{C}$, the location of the accessory pathway due to inspection of the conventional ECG recordings was supposed to be posteroseptal. The achieved inverse solution, however, could clearly reveal the accessory pathway to be located in the left posterior wall. This was also confirmed by the $\mathrm{CARTO}^{\mathrm{TM}}$ map. For the other patients the AT map corresponded to the findings from the conventional ECG and the CARTO ${ }^{\mathrm{TM}}$ map. The accessory pathway in patient $\mathrm{A}$ was located in a postero-septal position, in patient $\mathrm{B}$ and $\mathrm{D}$ the site was found to be in a left-lateral position. Figure 2 shows the on-line computed 

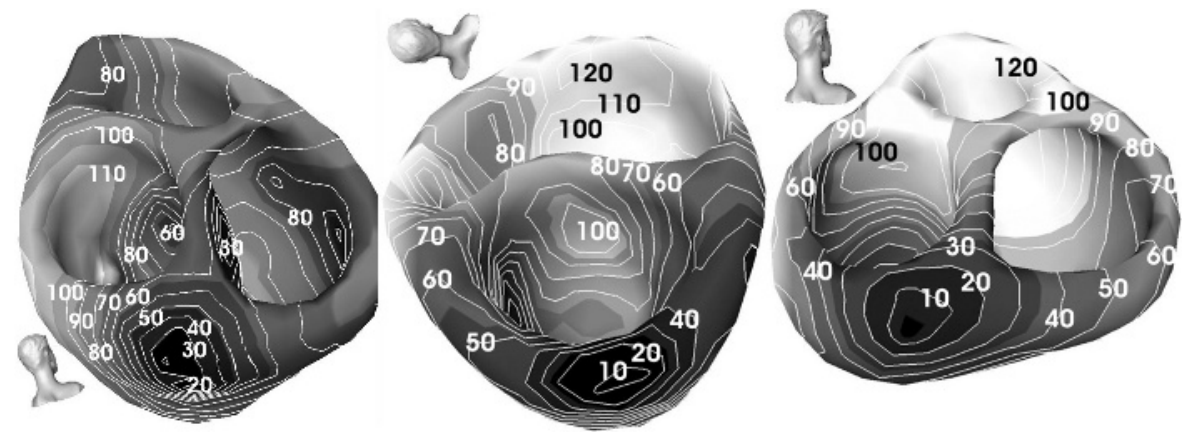

Fig. 2. Estimated electrical activation time sequences in patient A, B and C (left to right). Dark areas indicate early, white late activation. Isochrones are shown in steps of $10 \mathrm{~ms}$.

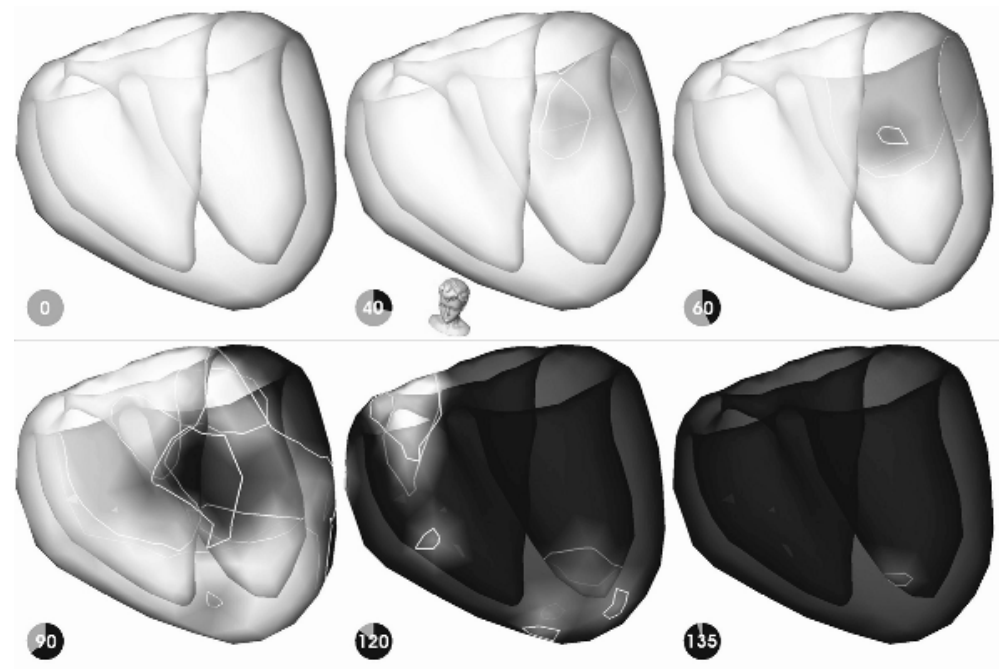

Fig. 3. Time series showing the spread of electrical activation in patient D. Dark areas indicate activated tissue, white areas tissue at resting potential. The isochrones show the site of the wavefronts. The accessory pathway was localized in a left-lateral position. The numbers in the small circles indicate the elapsed time in ms.

AT map of patients A, B and C. Figure 3 shows the time series of the calculated spread of electrical excitation in patient D.

After successful ablation of the accessory pathways, the 7 anatomical markers were digitized using the ablation catheter in the $\mathrm{CARTO}^{\mathrm{TM}}$ reference coordinate system. The transformation of the $\mathrm{CARTO}^{\mathrm{TM}}$ ablation points and anatomical markers into the MRI frame was done off-line. The location error between the 
successful ablation sites and the inversely found locations employing AT imaging could be determined between 13 and $20 \mathrm{~mm}$.

\section{Discussion and Conclusion}

Noninvasive imaging of the electrical activation was performed with four patients. The overall calculation time for these maps was in the same order as the time necessary for mapping the basal area of the ventricles using the CARTO ${ }^{\mathrm{TM}}$ system. With the AT imaging approach, the AT map of both chambers can be computed, whereas with the $\mathrm{CARTO}^{\mathrm{TM}}$ system only one or a part of one chamber can be imaged in the same time. Further enhancement of the noninvasive AT imaging approach can be achieved by:

- having anatomical imaging modalities (e.g., MRI) available in the EP lab,

- improving the techniques of semi-automatic segmentation of the electrical relevant compartments for generating the volume conductor model,

- automatic segmentation and classification of ECG signals and automatic determination of the target ECG waves.

Currently, the pipeline for segmenting the compartments of the volume conductor model and classification algorithms for detecting and classifying ECG target waves are under development.

This study clearly shows that on-line noninvasive AT imaging in the EP lab is feasible and supports the cardiologist with important information. In one case (patient C) AT imaging could locate the site of the accessory pathway more precisely compared to the presumed site based on conventional ECG data.

Further research will be about AT imaging for assessment of ablations in the right and left atrium of patients suffering from atrial flutter or fibrillation, as well as the imaging of atrial flutter circuits.

Acknowledgements. The authors would like to thank Sigrid Egger for her experienced technical support in the catheter laboratory.

This research study has been funded by the START Y144-N04 program granted by the Austrian Federal Ministry of Education, Science and Culture (bm:bwk) in collaboration with the Austrian Science Fund (FWF).

\section{References}

1. Lesh, M. D., Kalman, J. M., Olgin, J. E., Ellis, W. S.: The role of atrial anatomy in clinical atrial arrhythmias. J. Electrocardiol. 29 (1996) 101-113

2. Sippens-Groenewegen, A., Lesh, M.D., Roithinger, F.X., Ellis, W.S., Steiner, P. R., Saxon, L. A., Lee, R. J., Scheinman, M. M.: Body surface mapping of counterclockwise and clockwise typical atrial flutter: A comparative analysis with endocardial activation sequence mapping. J. Am Coll. Cardiol. 35 (2000) 1276-1287

3. Ben Haim, S. A., Osadchy, D., Schuster, I., Gepstein, L., Haya, G., Josephson, M. E.: Nonfluoroscopic, in vivo navigation and mapping technology. Nat. Med. 2 (1996) 1393-1395 
4. Fischer, G., Tilg, B., Modre, R., Huiskamp, G. J., Fetzer, J., Rucker, W., Wach, P.: A bidomain model based BEM-FEM coupling formulation for anisotropic cardiac tissue. Ann. Biomed. Eng. 28 (2000) 1229-1243

5. Greensite, F.: The mathematical basis for imaging cardiac electrical function. Crit. Rev. Biomed. Eng. 22 (1994) 347-399

6. Modre, R., Tilg, B., Fischer, G., Wach, P.: Noninvasive Myocardial Activation Time Imaging: A Novel Inverse Algorithm Applied to Clinical ECG Mapping Data. IEEE Trans. Biomed. Eng. 49 (2002) 1153-1161

7. Tilg, B., Fischer, G., Modre, R., Hanser, F., Messnarz, B., Schocke, M., Kremser, C., Berger, T., Hintringer, F., Roithinger, F. X.: Model-Based Imaging of Cardiac Electrical Excitation in Humans. IEEE Trans. Med. Imaging 21 (2002) 1031-1039

8. Geselowitz, D. B., Miller, III W. T.: A bidomain model for anisotropic cardiac muscle. Ann. Biomed. Eng. 11 (1983) 191-206

9. Henriquez, C.S.: Simulating the electrical behavior of cardiac tissue using the bidomain model. Crit. Rev. Biomed. Eng. 21 (1993) 1-77

10. Mosher, J. C., Leahy, R. M., Lewis, P.S.: EEG and MEG: Forward solutions for inverse methods. IEEE Trans. Biomed. Eng. 46 (1999) 245-259

11. Huiskamp, G.J., van Oosterom, A.: The depolarization sequence of the human heart surface computed from measured body surface potentials. IEEE Trans. Biomed. Eng. 35 (1988) 1047-1058

12. Oster, H.S., Taccardi, B., Lux, R. L., Ershler, P.R., Rudy, Y.: Noninvasive electrocardiographic imaging: Reconstruction of epicardia potentials, electrograms and isochrones and localization of single and multiple electrocardiac events. Circ. 96 (1997) 1012-1024

13. Modre, R., Tilg, B., Fischer, G., Hanser, F., Messnarz, B., Seger, M., Schocke, M. F. H., Berger, T., Hintringer, F., Roithinger, F. X.: Atrial Noninvasive Activation Mapping of Paced Rhythm Data. J. Cardiovasc. Electrophysiol. 14 (2003) $712-719$

14. Pfeifer, B., Hanser, F., Hintermüller, C., Modre, R., Fischer, G., Seger, M., Kremser, C., Tilg, B.: Atrial myocardium model extraction. Med. Imaging, Proceedings SPIE 2004 (2004) (in press)

15. Fischer, G., Tilg, B., Modre, R., Hanser, F., Messnarz, B., Wach, P.: On Modeling the Wilson terminal in the boundary and finite element method. IEEE Trans. Biomed. Eng. 49 (2002) 217-224

16. Huiskamp, G. J.: Difference formulas for the surface Laplacian on a triangulated surface. J. Comp. Phys. 95 (1991) 477-496

17. Hansen, P. C.: The L-curve and its use in the numerical treatment of inverse problems. In: Johnston, P. (Ed), Computational Inverse Problems in Electrocardiography, WIT Press, Southampton (2001) 119-142 